\title{
Array of biosensors for discrimination of grapes according to grape variety, vintage and ripeness
}

\author{
C. Medina-Plaza ${ }^{\text {a }}$, J.A. de Saja ${ }^{\mathrm{b}}$, J.A. Fernández-Escudero ${ }^{\mathrm{c}}, \mathrm{E}$ Barajas ${ }^{\mathrm{d}}$, G. Medrano ${ }^{\mathrm{e}}$, \\ M.L. Rodriguez-Mendez ${ }^{\text {a, * }}$
}

a Department of Inorganic Chemistry, Engineers School, Universidad de Valladolid, 47011 Valladolid, Spain

${ }^{\mathrm{b}}$ Department of Condensed Matter Physics, Faculty of Sciences, Universidad de Valladolid, 47011 Valladolid, Spain

${ }^{\mathrm{c}}$ Estacion Enologica de Castilla y Leon, Rueda, Spain

d ITACYL, Valladolid, Spain

e Bodega Cooperativa de Cigales, Valladolid, Spain

\section{H I G H L I G H T S}

- A bioelectronic tongue formed by biomimetic sensors has been developed.

- The system contains enzymes specific for the detection of phenols and sugars.

- The system is able to discriminate musts according with the grape variety.

- The loadings plot confirms the excellent complementarity of the biosensors.

- Excellent correlations with chemical analysis have been obtained.

\section{A R T I C L E I N F O}

\section{Article history:}

Received 8 March 2016

Received in revised form

20 June 2016

Accepted 19 October 2016

Available online 2 November 2016

\section{Keywords:}

Bioelectronic tongue

Grape

Langmuir-Blodgett

Enzyme

Bisphthalocyanine

Voltammetric sensor
G R A P H I C A L A B S T R A C T

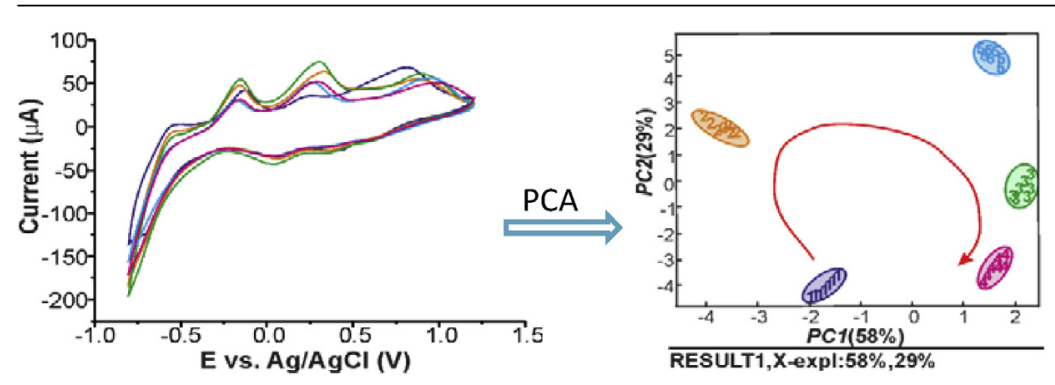

\begin{abstract}
A B S T R A C T
A bioelectronic tongue based on nanostructured biosensors specific for the simultaneous detection of sugars and phenols has been developed. The array combined oxidases and dehydrogenases immobilized on a lipidic layer prepared using the Langmuir-Blodgett technique where Glucose oxidase, D-Fructose dehydrogenase, Tyrosinase or Laccase were imbibed. A phthalocyanine was co-immobilized in the sensing layer and used as electron mediator. The array thus formed has been used to analyze grapes and provides global information about the samples while providing specific information about their phenolic and their sugar content. Using Principal Component Analysis (PCA) the array of voltammetric biosensors has been successfully used to discriminate musts prepared from different varieties of grapes (Tempranillo, Garnacha, Cabernet-Sauvignon, Prieto Picudo and Mencía). Differences could be also detected between grapes of the same variety and cultivar harvested in two successive vintages (2012 and 2013).

Moreover, the ripening of grapes could be monitored from veraison to maturity due to the changes in their phenolic and sugar content. Using Partial Least Squares (PLS-1) analysis, excellent correlations have been found between the responses provided by the array of biosensors and classical parameters directly related to phenols (total polyphenol index, TPI) and sugar concentration (degree Brix) measured by chemical methods with correlation coefficients close to 1 and errors close to 0 . It is also worthy to notice the good correlations found with parameters associated with the $\mathrm{pH}$ and acidity that can be explained by
\end{abstract}

\footnotetext{
* Corresponding author. Dpt. Inorganic Chemistry, Escuela de Ingenierías Indus-
} triales, Paseo del Cauce, 59, 47011 Valladolid, Spain.

E-mail address: mluz@eii.uva.es (M.L. Rodriguez-Mendez). 
taking into account the influence of the $\mathrm{pH}$ in the oxidation potentials of the phenols and in the enzymatic activity.

This bioelectronic tongue can assess simultaneously the sugar and the phenolic content of grapes and could be used to monitor the maturity of the fruit and could be adapted easily to field analysis.

() 2016 Elsevier B.V. All rights reserved.

\section{Introduction}

The quality of the grapes is typically established on the basis of their phenolic and sugar content [1,2]. Sugar content is the parameter usually used to monitor the maturity of grapes because it is directly associated with the alcohol degree of the final product. Phenols are a large group of metabolites with a direct influence on the organoleptic characteristics of wine [3]. There is an increasing interest to detect the phenolic maturity of grapes, a parameter that would be crucial to decide when to harvest.

Sugar and phenolic content of wines and musts are usually analyzed using optical, spectroscopic or chromatographic methods [4]. Electrochemical biosensors can be an alternative to classical methods due to their high specificity, low detection limits, rapid response and possibility of miniaturization $[5,6]$.

A large variety of biosensors dedicated to the detection and quantification of phenols has been developed. Those sensors contain phenol oxidases such as tyrosinase, laccase or horseradish peroxidase [7]. Similarly, many efforts have been carried out to develop biosensors devoted to the detection of sugars using glucose oxidase $[8,9]$. Biosensors containing other enzymes such as fructose dehydrogenase have been studied in lesser extent [10,11].

The immobilization of the enzyme onto the substrate is a key step on the development of efficient biosensors with high enzymatic activity [12]. Classical immobilization strategies include adsorption on a substrate [13], immobilization in a carbon paste or PVC matrix [14,15], entrapment in a polymeric matrix [16], covalent binding [17], cross-linking [18] or encapsulation on liposomes among many others [19]. The advances in Nanoscience and Nanotechnology provide alternative methods to immobilize biomolecules preserving their activity more efficiently. Layer by layer (LbL) [20-22] and Langmuir-Blodgett (LB) [23-25] are two examples of these techniques. The advantages of LbL and LB films are directly associated with the control of the thickness and the possibility of tuning the molecular architectures. Moreover, these techniques allow preparing sensors where the enzymes are adsorbed in biomimetic lipidic layers formed by fatty acids or phospholipids (similar to membrane cells) via $\mathrm{COOH}$ group interaction. This biomimetic environment contributes to the preservation of the enzymatic conformation. This strategy has been successfully used to prepare glucose oxidase [26] and tyrosinase biosensors [27]. Up to now LB films of dehydrogenases have not been developed. LB technique can also be advantageous because the electron mediator can be co-immobilized with the enzyme in the lipidic layer, facilitating the electron transfer process [27-29].

During the last years, multisensory systems combined with appropriate pattern recognition software (electronic tongues) have been developed and applied to analyze complex liquids [30,31]. They have been successfully used for the discrimination and classification of wines according to their grape variety, type of ageing or adulteration [32-34] and grapes [35].

It has been claimed that arrays formed by biosensors can be advantageous because they combine the advantages of classical arrays of electrochemical sensors (that provide global information about the sample) with the specificity of the enzyme-substrate reaction typical of biosensors. In spite of their good capability to discriminate wines or beers, bioelectronic tongues have rarely been used to obtain specific information about the composition of the samples $[14,36,37]$.

Nowadays, the quality of grapes is only analyzed by measuring the sugar content. There is a need to monitor simultaneously the phenolic maturity. Unfortunately, up to now there is not a method able to monitor simultaneously the sugar and phenolic content. The aim of this work was to develop an array of nanostructured biosensors (a bioelectronic tongue) able to discriminate musts prepared from different varieties of grapes, to discriminate grapes harvested in two different vintages and to monitor the ripening of grapes from veraison to harvest according to their sugar and phenolic content. For this purpose a multisensory system formed by four LB films containing two phenol oxidases (tyrosinase and laccase) and two sensors dedicated to the detection of sugars (glucose oxidase and D-fructose dehydrogenase) has been developed. This is the first time that oxidases and dehydrogenases have been combined. The capability of discrimination of the array has been evaluated using Principal Component Analysis (PCA). The correlation of the responses with the phenolic and sugar content of the grapes analyzed by classical chemical methods has been evaluated using Partial Least Squares (PLS-1).

\section{Materials and methods}

\subsection{Chemicals}

Laccase (Lac), from Trametes versicolor (EC Number: 1.10.3.2, activity of plus of $20.7 \mathrm{U} \mathrm{mg}^{-1}$ ), Tyrosinase (Tyr) from Mushroom (EC Number 1 1.14.18.1, activity of $3610 \mathrm{U} \mathrm{mg}^{-1}$ ), Glucose Oxidase (GOX) from Aspergillus Niger, type VII (EC Number: 1.1.3.4, activity plus $100000 \mathrm{U} \mathrm{mg}^{-1}$ ) and D-Fructose Dehydrogenase (FDH) from Gluconobacter Industrius (EC Number:1.1.99.11, activity of 400-1200 $\mathrm{U} \mathrm{mg}^{-1}$ ) were purchased from Sigma Chemical Co. (USA). $70 \mu \mathrm{g} \mathrm{mL} \mathrm{m}^{-1}$ solutions of the phenol oxidases were prepared in phosphate buffer $0.01 \mathrm{~mol} \mathrm{~L}^{-1}(\mathrm{pH}=7.0)(\mathrm{PBS})$ whereas solution of glucose oxidase and $\mathrm{D}$-fructose dehydrogenase were prepared in phosphate buffer $0.01 \mathrm{~mol} \mathrm{~L}^{-1}(\mathrm{pH}=4.5)$ (PBS).

All chemical and solvents (Sigma Chemical Ltd.) were of reagent grade and used as supplied. Deionized water was obtained from a Millipore purifier $\left(18.2 \mathrm{M} \Omega \mathrm{cm}^{-1}\right.$ ).

The lutetium (III) bisphthalocyaninate was synthesized and purified in the neutral radical state following a previously published procedure [38].

\subsection{Grape samples}

Five varieties of grapes (Tempranillo, Garnacha, CabernetSauvignon, Prieto Picudo and Mencía) were selected and harvested in Castilla y León region (Spain). Grapes were harvested from the same cultivar in two consecutive vintages (2012 and 2013). In addition, during the year 2013, grapes of Tempranillo variety were collected periodically in order to monitor the ripening. Sample 1 corresponded to grapes collected at veraison (change of the grape 
color from green to red). Then, samples were collected on a weekly basis (Samples 2 to 5). To obtain the musts, $300 \mathrm{~g}$ of each type of grape were crushed using standard procedures. This process was carried out by septuplicate giving a total of 98 samples. Chemical analyses were carried out following international regulations [4]. Parameters measured included classical chemical markers of the sugar content (Degree brix, Density, Sugars, Grade (16.8), Grade (17.5)), markers of the polyphenolic content (Total Polyphenol Index, TPI) and indicators of the acidity ( $\mathrm{pH}$, Total acidity, Malic acid, Tartaric acid).

\subsection{Langmuir-Blodgett films}

LB sensors containing enzyme (Enz), fatty acid (arachidic acid, $\mathrm{AA}$ ) and the electron mediator (lutetium bisphthalocyanine, $\mathrm{LuPc}_{2}$ ) (Enz/AA/LuPc 2 ), were fabricated using a KSV 2000 trough (KSV Instruments, Finland). Films of Tyr/AA/LuPc 2 and $\mathrm{Lac} / \mathrm{AA} / \mathrm{LuPc}_{2}$ were prepared using the conditions previously described [33]. LB film sensors based on FDH and GOX are reported here for the first time and preparation conditions were established in order to maximize the insertion of the enzyme into the floating film.

GOX and FDH films were prepared at $\mathrm{pH} 4.5$ using a phosphate buffered saline (PBS-NaCl) subphase $\left(\mathrm{NaCl} 0.1 \mathrm{~mol} \mathrm{~L}^{-1}\right.$, phosphate buffer $\left.0.01 \mathrm{~mol} \mathrm{~L}^{-1}, \mathrm{pH}=4.5\right)$. Langmuir monolayers at the air/ water interface were characterized by surface pressure vs. mean molecular area $(\pi-\mathrm{A})$ isotherms at $21^{\circ} \mathrm{C}$ by spreading $100 \mu \mathrm{l}$ of a chloroform AA/LuPc 2 mixture (10:1) onto the subphase. Once the solvent was evaporated, $40 \mu \mathrm{l}$ of a $70 \mu \mathrm{g} \mathrm{ml}^{-1}$ solution of the corresponding enzyme (GOX or $\mathrm{FDH})$ in $0.01 \mathrm{~mol} \mathrm{~L}^{-1} \mathrm{PBS}(\mathrm{pH}=4.5)$ were injected drop by drop underneath the air/liquid interface. The enzymes were then adsorbed inside the floating film for one hour. Barriers were compressed at a speed of $5 \mathrm{~mm} \mathrm{~min}^{-1}$.

$\mathrm{Enz} / \mathrm{AA} / \mathrm{LuPc}_{2}$ transferred to ITO glass at a surface pressure of $40 \mathrm{mN} \mathrm{m}^{-1}$ by Y-type deposition with a transfer ratio close to 1 with a substrate speed of $3 \mathrm{~mm} \mathrm{~min}^{-1}$. Finally, the Enz/AA/LuPc 2 LB films were treated with glutaraldehyde (2.5\% w/v in PBS).

Brewster Angle Microscope (BAM) images were registered using a KSV/Nima MicroBAM.

\subsection{Electrochemical measurements}

Biosensors were used as working electrode, the reference electrode was $\mathrm{Ag} \mid \mathrm{AgCl} / \mathrm{KCl} 3 \mathrm{M}$ and the counter electrode was a platinum plate. The potentiostat was an EG\&G PARSTAT 2273 potentiostat/galvanostat. Cyclic voltammograms were registered at a sweep rate of $0.1 \mathrm{~V} \mathrm{~s}^{-1}$ from $-0.8 \mathrm{~V}$ to $+1.0 \mathrm{~V}$. In order to facilitate the diffusion of ions and to reduce the complexity of the sample, musts were diluted $50 \%$ in water.

\subsection{Statistical analysis}

Curves were pre-processed a data reduction technique based on "kernels" [39]. The voltammogram curve is multiplied by 10 smooth, bell-shaped windowing functions defined as:

$K_{i}\left(V_{j}\right)=\frac{1}{1+\left(\frac{V_{j}-c_{i}}{a_{i}}\right)^{2 b_{i}}}$

where $a_{i}, b_{i}$ and $c_{i}$ define the width, shape and center of the different windowing functions $K_{i}$. Subsequently, data were integrated with respect to voltage. After compression, each voltammogram has been reduced to a vector of 10 variables which were used as input data source for statistical analysis.

A non-supervised multivariate method, Principal Component
Analysis (PCA), was used to evaluate the capability of discrimination of the system. Partial Least Squares (PLS-1) method was used to establish correlations between the signals produced by the array of biosensors and the results obtained by means of chemical methods. Chemometric analyses were carried out using the software Matlab v5.3. (The Mathworks Inc., Natick, MA, USA).

\section{Results and discussion}

\subsection{Characterization of the Langmuir films}

In this work, novel sensors have been developed based on Glucose Oxidase (GOX) and D-Fructose Dehydrogenase (FDH). They have been combined with biosensors containing tyrosinase and laccase. This, four biosensors will form an array containing enzymes able to detect phenols and sugars, two of the most important parameters to be analyzed in grapes.

The first step in this work was to develop a method to prepare GOX/AA/LuPc 2 and FDH/AA/LuPc 2 LB films. For this purpose, Surface pressure/Area $(\pi / \mathrm{A})$ isotherms of the $\mathrm{Enz} / \mathrm{AA} / \mathrm{LuPc}_{2}$ systems were registered. Fig. 1 shows the isotherms registered for the four sensing layers included in the array. The isotherm obtained from the mixture of the lipid and the electron mediator $\left(\mathrm{AA} / \mathrm{LuPc}_{2}\right)$ has been included in the graph for comparison purposes.

The limiting area obtained from the mixture $\mathrm{AA} / \mathrm{LuPc}_{2}$ was $37 \AA^{2}$. As expected, the incorporation of enzymes produced the expansion of the isotherms to higher areas per molecule.

The limiting area per molecule values obtained for GOX/AA/ $\mathrm{LuPc}_{2}$ and $\mathrm{FDH} / \mathrm{AA} / \mathrm{LuPc}_{2}$ with values of $63 \mathrm{mN} \mathrm{m}^{-1}$ and $60 \mathrm{mN} \mathrm{m}^{-1}$ were clearly higher than those observed for phenol oxidases ( $47 \mathrm{mN} \mathrm{m}^{-1}$ for Lac/AA/LuPc 2 and $51 \mathrm{mN} \mathrm{m}^{-1}$ for Tyr/AA/LuPc 2 ). This indicated that enzymes were adsorbed in the amphiphilic monolayer. The large values observed in GOX and FDH can be due to an enhanced adsorption of the enzyme into the AA environment caused by more favorable hydrophobic-hydrophilic interactions between the enzymes and the floating $A A / L P_{2}$ film. The differences in molecular weight of the enzymes must also have an important effect (120-133 KDa for Tyr, 50-100 KDa for Lac, $160 \mathrm{KDa}$ for GOX, $150 \mathrm{KDa}$ for FDH). In fact, GOX and FDH which present the higher molecular weights also show the larger areas per molecule.

In order to better understand the intermolecular interactions inside the floating films, compression-expansion cycles were analyzed (Fig. 1b and c). Under these conditions, the behavior of GOX and FDH enzymes was different from the behavior shown by phenol oxidases which are desorbed during the expansion cycle [40]. In the case of GOX/AA/LuPc 2 and $\mathrm{FDH} / \mathrm{AA} / \mathrm{LuPc}_{2}$ the limiting area per molecule obtained after decompression was only slightly smaller than the value observed in the first compression. During the second compression, the area per molecule values were only slightly smaller than those registered in the first compression. In summary, only a small amount of GOX or FDH was desorbed from the film during the expansion. In addition, a large part of this desorbed enzyme was readsorbed during the second compression. Because GOX and FDH are not completely expelled from the film during decompression and are easily readsorbed it can be concluded that the interactions with the $\mathrm{AA} / \mathrm{LuPc}_{2}$ film are stronger than in the case of Tyr and Lac, justifying the larger area per molecule observed.

The homogeneity of the floating films was analyzed using Brewster Angle Microscopy (BAM) (Fig. 1b and $1 \mathrm{c}$ inset). Good quality surfaces were observed for all the films analyzed. BAM images also showed a clear increase in the roughness of the film (with respect to the $\mathrm{AA} / \mathrm{LuPc}_{2}$ film). This increase was particularly evident in GOX/AA/LuPc 2 films that could be associated with the 

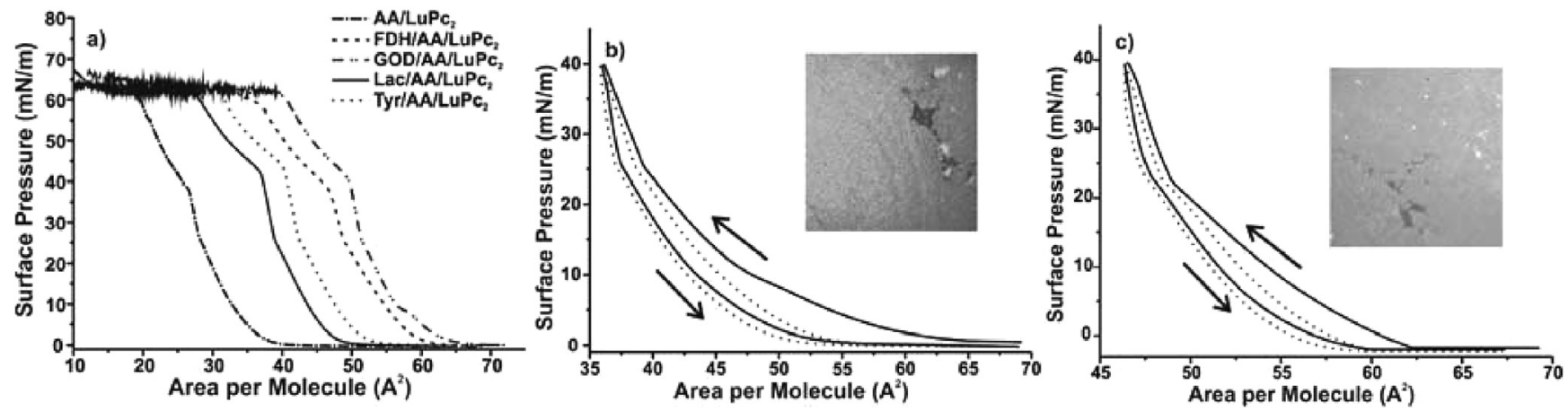

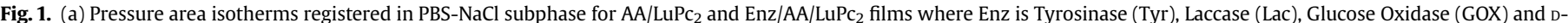

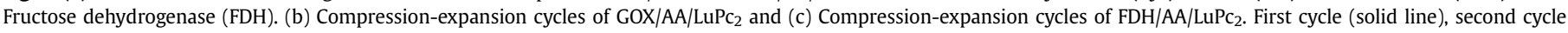
(dotted line). Inset show BAM images.

high affinity of the enzyme towards the lipidic layer.

\subsection{Characterization of the biosensors}

The individual response of the biosensors was previously tested towards primary substrate solutions (D-glucose, D-fructose and catechol) and the limits of detection were calculated as $3 \mathrm{SD} / \mathrm{b}$ (where SD is the standard deviation of the blank and $b$ is the slope of the calibration graph). The limits of detection found were 0.46 , $0.52,77$ and $9 \mu \mathrm{M}$ for tyrosinase, laccase, glucose oxidase and Dfructose dehydrogenase respectively. The voltammetric responses obtained were highly reproducible with a coefficient of variation $(n=10)$ lower than $2 \%$ calculated from the peaks associated with the analytes. The reproducibility between different sensors (i.e. containing the same enzyme) immersed on the same solution was always lower than $2.5 \%$. All the measurements present associated uncertainties lower than $5 \%$.

It is important to remark that sensors are highly reproducible and this is a key feature to be able to monitor the ripening of grapes for months.

\subsection{Discrimination of musts obtained from different varieties of grapes harvested in two consecutive vintages}

The array formed by four biosensors was used to analyze musts prepared from five varieties of grapes (Tempranillo, Garnacha, Cabernet-Sauvignon, Prieto Picudo and Mencía) harvested in two different vintages (2012 and 2103) from the same cultivar.

The response of the array is illustrated in Fig. 2 for Prieto Picudo variety. The peaks related to the antioxidants appear as anodic waves in the $0.5-1.0 \mathrm{~V}$ region with the corresponding cathodic peaks at $c a .0 .6 \mathrm{~V}$. The peaks associated with sugars appeared at negative potentials $(\mathrm{ca} .-0.2 \mathrm{~V})$. Obviously, due the complexity of the media, the peaks were wider than those observed in standard aqueous solutions [41]. Each electrode provided a different response towards the same must sample and the positions and the intensity of the peaks changed with the grape variety. Moreover, voltammograms could detect small differences from one vintage to another however, the general behavior of grapes of the same variety was maintained.

When PCA was carried out in musts obtained from grapes of different varieties (harvested in 2012), the scores plot showed that the array was able to discriminate musts according to the type of grape. The three first components explained $\mathrm{ca}$. $97 \%$ of the total information of the system.

The experiment was repeated in the vintage 2013 and the results were similar. Moreover, PCA was carried out merging in the

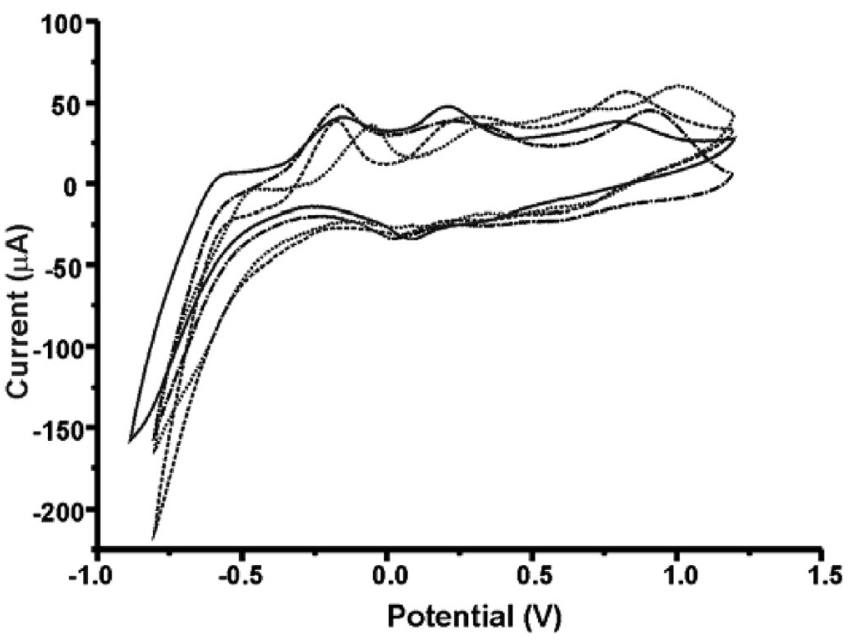

Fig. 2. Voltammetric responses of the four sensors towards must prepared from the grape variety Prieto Picudo harvested in 2012. Glucose oxidase (black solid line), Laccase (dotted line), D-Fructose dehydrogenase (dash-dot line) and Tyrosinase (dashed line).

same matrix the responses obtained in both 2012 and 2013 (Fig. 3). Grapes were discriminated according to their variety. Samples collected in different vintages could be clearly distinguished but appeared close one to each other in the scores plot. This result indicates that the bioelectronic tongue provides similar results than classical chemical analyses that clearly establish that varietal qualities are maintained from one year to another.

The position of the clusters was directly related to the chemical composition of the grapes. For this reason, the must prepared from Mencía variety, with the lowest concentration of sugar (average of $215 \mathrm{~g} \mathrm{~L}^{-1}$ ) and lower TPI (average of 12), was located in the left side of the diagram, whereas samples from Tempranillo variety with the highest sugar content (average of $238.2 \mathrm{~g} \mathrm{~L}^{-1}$ ) and highest TPI (average of 20) were located in the top part of the figure. Musts obtained from Garnacha, Cabernet-Sauvignon and Prieto Picudo grapes, with intermediate sugar $\left(220-230 \mathrm{~g} \mathrm{~L}^{-1}\right)$ and TPI values (TPI of 15), appeared far apart from the rest. pH seems to play an important role in the response of the array: Tempranillo and Mencía samples showed the highest pHs (4.18 and 4.17 respectively) and were located far from Prieto Picudo (3.7) and CabernetSauvignon (3.8). Finally, Garnacha which showed the lowest $\mathrm{pH}$ (3.4) was clearly discriminated from the rest.

Fig. 3 b shows the contribution of the variables ( 10 kernels per sensor) in the PCA loading plot. The loadings of each sensor were 


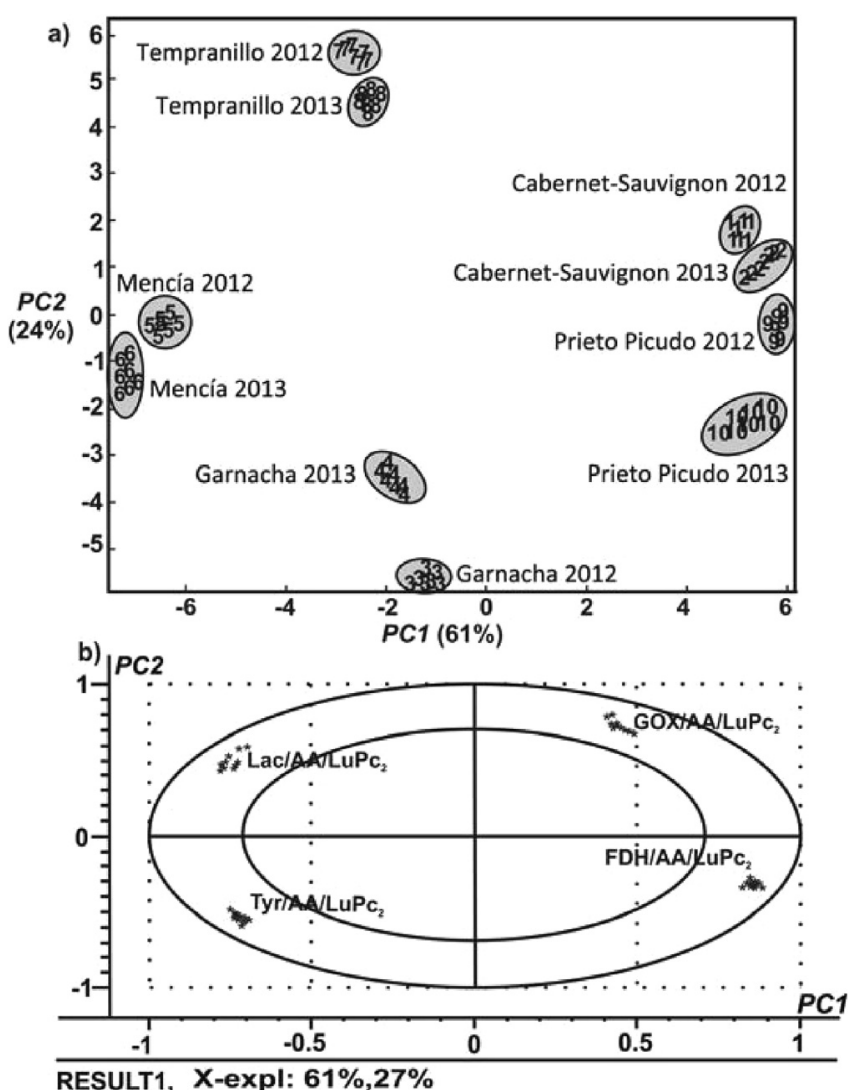

Fig. 3. PCA scores plot (up) and loading plot (down) illustrating the discrimination capability of the bioelectronic tongue exposed to must prepared from the same five varieties harvested in two vintages (2012 and 2013).

located in a different quadrant and showed values close to 1 . This is important because each one of the four sensors forming the array contributes in an important manner to the discrimination process. This also evidences the high cross-selectivity attained by the four biosensors.

\subsection{Monitoring the grape ripeness by means of the bioelectronic tongue}

The bioelectronic tongue was used to analyze grapes harvested from the veraison to ripening in a weekly basis during five weeks (variety Tempranillo, vintage 2013). Sample 1 was collected during the veraison (change of the grape color from green to red). Samples 2-5 were harvested in subsequent weeks. Sample number 4 corresponded to the optimal date of harvesting predicted by chemical parameters. Sample 5 corresponded to over-ripened grapes.

Fig. 4 illustrates how voltammograms changed with the ripening using a tyrosinase electrode. The anodic peak at $c a .0 .7 \mathrm{~V}$ associated with polyphenols shifted to higher potentials whereas the peaks at $c a$. $0.4 \mathrm{~V}$ and $-0.1 \mathrm{~V}$ increased their intensity progressively from weeks $1-3$. Suddenly, in week 4 (the optimal date as indicated by classical techniques) the intensity decreased and was maintained in over-ripened grapes collected in week 5. These changes are in good agreement with changes in the phenolic maturation measured by chemical methods which indicate that the development of phenolic maturation in the late stage of ripening produces a notorious increase near the harvest [42]. Similar results were obtained with biosensors modified with laccase. In turn, sensors modified with glucose oxidase could follow the increase in

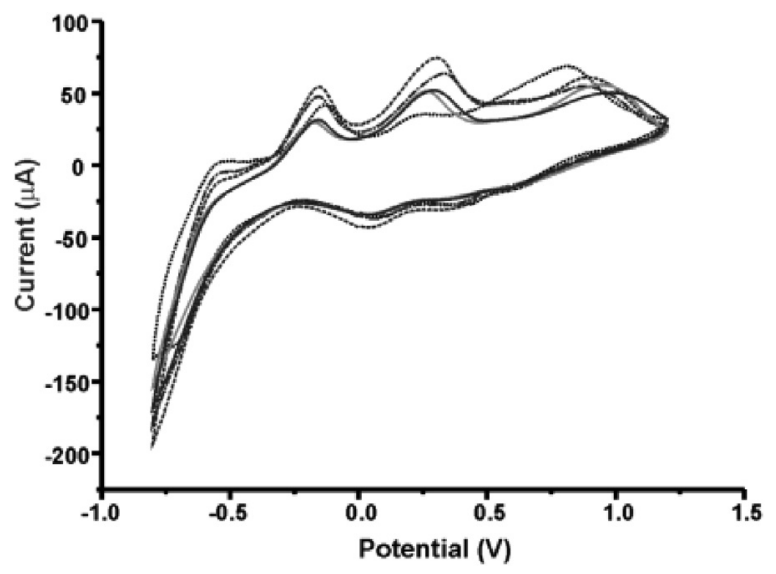

Fig. 4. Response of the $\mathrm{Tyr} / \mathrm{AA} / \mathrm{LuPc}_{2}$ towards all the samples of Tempranillo harvested weekly from veraison to over-ripeness (vintage 2013). First week (dotted line), second week (dash-dot line), third week (dashed line), fourth week (grey solid line) and fifth week (black solid line).

the sugar content by showing an increase in the intensity of the cathodic peak at $-0.2 \mathrm{~V}$.

PCA showed a clear discrimination of the samples collected during the progression of maturing. (Fig. 5). Clusters corresponding to samples harvested in successive weeks from veraison to ripening (samples 1 to 4 ) were located in a clockwise order. Grapes collected one week after the optimal maturity (sample 5), did not follow the same trend than the others.

\subsection{Multiparametric correlations between the bioelectronic tongue and the classical chemical analysis}

Table 1 shows the statistical parameters for the PLS-1 regression models correlating the output of the array with the chemical parameters. In order to select the number of factors a leave-one-out cross-validation method was used. This method involves using one observation as the validation set and the remaining (n-1) observations as the training set. This is repeated on all ways to cut the

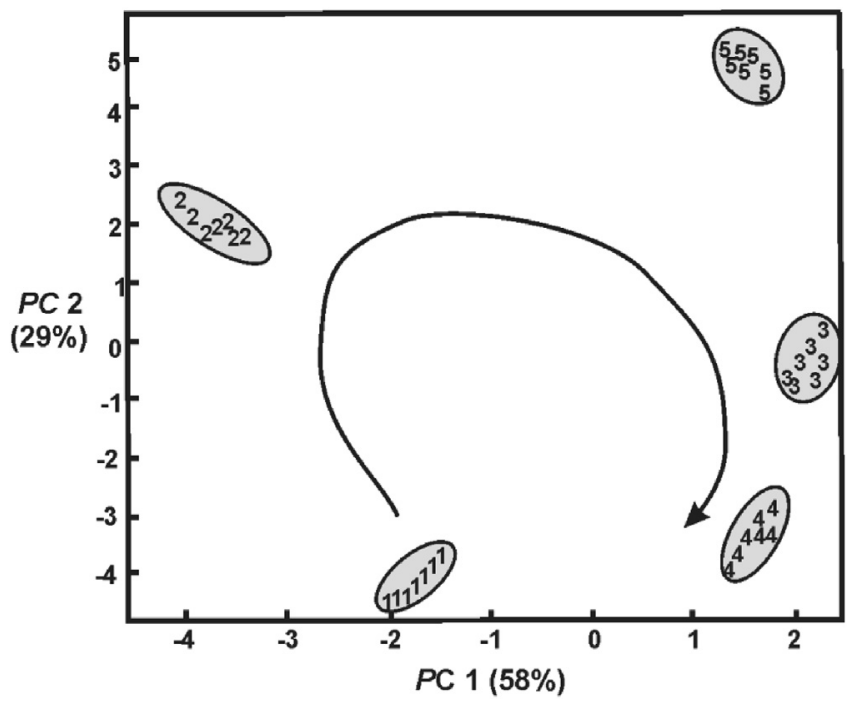

Fig. 5. PCA scores plot illustrating the discrimination capability of the bioelectronic tongue exposed to must prepared from Tempranillo samples harvested in 2013 weekly from 1-first week, 2-s week, 3-third week, 4-fourth week (harvest), 5-fifth week (overripened). 
Table 1

Results of the PLS-1 analysis.

\begin{tabular}{llllll}
\hline Parameter & RMSEC $^{\mathrm{a}}$ & RMESP $^{\mathrm{b}}$ & $\mathrm{R}_{\mathrm{C}}{ }^{\mathrm{c}}$ & $\mathrm{R}_{\mathrm{P}}{ }^{\mathrm{d}}$ & $\mathrm{LV}^{\mathrm{e}}$ \\
\hline Degree brix & 0.25352 & 0.30653 & 0.9874 & 0.9818 & 3 \\
Density & 0.00309 & 0.00502 & 0.9042 & 0.7319 & 2 \\
Sugars & 0.80089 & 1.29796 & 0.9024 & 0.7285 & 2 \\
Grade (16.8) & 0.47520 & 0.77026 & 0.9024 & 0.7288 & 2 \\
Grade (17.5) & 0.45787 & 0.73801 & 0.9002 & 0.7249 & 2 \\
pH & 0.05800 & 0.06997 & 0.9822 & 0.9740 & 3 \\
Total acidity & 0.04724 & 0.05948 & 0.9948 & 0.9918 & 3 \\
Malic acid & 0.20872 & 0.24564 & 0.9266 & 0.8973 & 3 \\
Tartaric acid & 0.12617 & 0.14325 & 0.9955 & 0.9943 & 3 \\
TPI & 0.59340 & 0.67736 & 0.9731 & 0.9649 & 3 \\
\hline
\end{tabular}

${ }^{a}$ Root Mean Square Error of Calibration.

b Root Mean Square Error of Prediction.

c Squared correlation coefficient in calibration.

d Squared correlation coefficient in prediction.

e Latent variables.

original sample on a validation set of one observation and n-1 training set.

Excellent correlations were found for all the parameters related to sugar (sugar, degree brix, grade and density) and in particular with the degree Brix which is commonly used to evaluate the quality of grapes. Also good correlations have been found with TPI. These good correlations can be attributed to the excellent performance of the enzymes imbibed in the LB films. It is also worthy to notice the good correlations found with parameters related to the $\mathrm{pH}(\mathrm{pH}$, Total acidity, Malic and Tartaric acid). This can be explained by the influence of the $\mathrm{pH}$ in the enzymatic activity.

\section{Conclusions}

A bioelectronic tongue formed by four voltammetric biosensors based on LB films containing glucose oxidase, D-fructose dehydrogenase, tyrosinase and laccase, using $\mathrm{LuPc}_{2}$ as electron mediator and AA as lipid, has been developed. The increase in the area per molecule observed in the surface pressure-area isotherms confirms that enzymes are imbibed inside the floating monolayer formed by $\mathrm{LuPC}_{2}$ and $\mathrm{AA}$.

The array of biosensors is able to discriminate five varieties of grapes according to their sugar and phenolic composition. The system is more sensitive to the differences caused by the variety of grape than to the changes caused by weather conditions from vintage to vintage. It is also able to monitor the ripening of grapes from veraison to harvesting and could be used to predict the appropriate date to harvest.

The loading plot confirms the excellent complementarity of the biosensors. Moreover, PLS-1 shows exceptional correlations with all the chemical parameters directly related to phenols and sugar concentration.

\section{Acknowledgments}

Financial support by the MINECO and FEDER (grant CICYTAGL2012-33535) and Junta Castilla y León (VA-032U13) is gratefully acknowledged. CMP thanks the University of Valladolid for the grant (PIF-UVa).

\section{References}

[1] R. Boulton, The copigmentation of anthocyanins and its role in the color of red wine: a critical review, Am. J. Enol. Vitic. 52 (2001) 67.

[2] Y. Glories, Caractérisation du potentiel phénoliqueadaptation de la vinification, Progrès Agric. Vitic. 118 (2001) 347.

[3] B.S. Sun, T. Pinto, M.C. Leandro, J.M. Ricardo-Da-Silva, M.I. Spranger, Transfer of catechins and proanthocyanidins from solid parts of the grape cluster into wine, Am. J. Enol. Vitic. 50 (1999) 179.

[4] OIV, Compendium of International Methods of Analysis of Wines and Musts, 2 2013.

[5] A. Sassolas, L.J. Blum, B.D. Leca-Bouvier, Immobilization strategies to develop enzymatic biosensors, Biotechnol. Adv. 90 (2012) 489.

[6] D.R. Thevenot, K. Toth, R.A. Durst, G.S. Wilson, Electrochemical biosensors: recommended definitions and classification, Biosens. Bioelectron. 16 (2001) 121.

[7] K. Fahmida, A.N.M. Fakhruddin, Recent advances in the development of biosensor for phenol: a review, Rev. Environ. Sci. Biotech. 11 (2012) 261.

[8] W. Nouira, A. Maaref, H. Elaissari, F. Vocanson, M. Siadat, N. Jaffrezic-Renault, Comparative study of conductometric glucose biosensor based on gold and on magnetic nanoparticles, Mater. Sci. Eng. C 33 (2013) 298.

[9] M.A. Kamyabi, N. Hajari, A.P.F. Turner, A. Tiwari, A high-performance glucose biosensor using covalently immobilised glucose oxidase on a poly(2,6diaminopyridine)/carbon nanotube electrode, Talanta 116 (2013) 801.

[10] R. Antiochina, G. Vinci, L. Gorton, Rapid and direct determination of fructose in food: a new osmium-polymer mediated biosensor, Food Chem. 140 (2013) 742.

[11] K. Damar, D.O. Demirkol, Modified gold surfaces by poly(amidoamine) dendrimers and fructose dehydrogenase for mediated fructose sensing, Talanta 87 (2011) 67.

[12] T.E. Goto, R.F. Lopez, O.N. Oliveira, L. Caseli, Enzyme activity of catalase immobilized in Langmuir-Blodgett films of phospholipids, Langmuir 26 (2010) 11135.

[13] M. Moyo, J. Okonkwo, Horseradish peroxidase biosensor based on maize tassel-MWCNTs composite for cadmium detection, Sens. Actuators B 193 (2014) 515.

[14] X. Cetó, J. Capdevilla, S. Minguez, M. del Valle, Voltammetric BioElectronic Tongue for the analysis of phenolic compounds in rosé cava wines, Food Res. Int. 55 (2014) 455.

[15] I.M. Apetrei, M.L. Rodriguez-Mendez, C. Apetrei, J.A. de Saja, Enzyme sensor based on carbon nanotubes/cobalt(II) phthalocyanine and tyrosinase used in pharmaceutical analysis, Sens. Actuators B 177 (2013) 138.

[16] A.M. Mariani, M.E. Natoli, P. Kofinas, Enzymatic activity preservation and protection through entrapment within degradable hydrogels, Biotechnol. Bioeng. 10 (2013) 2994.

[17] J. Dong, T. Liu, X. Meng, J. Zhu, K. Hang, S. Ai, S. Cui, Amperometric biosensor based on immobilization of acetylcholinesterase via specific binding on biocompatible boronic acid-functionalized Fe@Au magnetic nanoparticles, J. Solid State Electrochem. 16 (2012) 3783.

[18] F.C. Vincentini, B.C. Janegitz, C.M.A. Brett, O. Fatibello-Filho, Tyrosinase biosensor based on a glassy carbon electrode modified with multi-walled carbon nanotubes and1-butyl-3-methylimidazolium chloride within adihexadecylphosphate film, Sens. Actuators B 188 (2013) 1101.

[19] H. Guan, X. Liu, W. Wang, Encapsulation of tyrosinase within liposome bioreactors for developing an amperometric phenolic compounds biosensor, J. Solid State Electrochem. 17 (2013) 2887.

[20] M.L. Ho, J.C. Wang, T.Y. Wang, C.Y. Lin, J.F. Zhu, Y.A. Chen, T.C. Chen, The construction of glucose biosensor based on crystalline iridium(III)-containing coordination polymers with fiber-optic detection, Sens. Actuators B 190 (2014) 479.

[21] V. dos Santos, M. dos Santos, C.G. de Jesus, S.T. Fujiwara, J.R. Garcia, C.A. Pessoa, K. Wohrath, The role of a layer-by-layer film containing Pt nanoparticle on the performance of a glucose enzymatic biosensor, Int. J. Electrochem. Sci. 8 (2013) 10601.

[22] E.G.R. Fernandes, L.C. Brazaca, M.L. Rodriguez-Mendez, J.A. de Saja, V. Zucolotto, Immobilization of lutetium bisphthalocyanine in nanostructured biomimetic sensors using the LbL technique for phenol detection, Biosens. Bioelectron. 26 (2011) 4715 .

[23] J. Cabaj, J. Soloducho, A. Nowakowska-Oleksy, Immobilization of uricase enzyme in Langmuir and Langmuir-Blodgett films of fatty acids: possible use as a uric acid sensor, Sens. Actuators B 143 (2010) 508.

[24] N.C.M. Zanon, O.N. Oliveira Jr., L. Caseli, Langmuir-Blodgett film based biosensor for estimation of phenol derivatives, J. Colloid Interface Sci. 373 (2012) 69.

[25] L. Challier, F. Gal, G. Carrot, H. Perez, V. Noel, Hybrid platinum nanoparticle ensemble for the electrocatalytic oxidation of H2O2: toward nanostructured biosensor design, Electrochem. Commun. 28 (2013) 118.

[26] K.H. Wang, M.J. Syu, C.H. Chang, Y.L. Lee, Immobilization of glucose oxidase by Langmuir-Blodgett technique for fabrication of glucose biosensors: headgroup effects of template monolayers, Sens. Actuators B 164 (2012) 29.

[27] F.J. Pavinatto, E.G.R. Fernandes, P. Alessio, C.J.L. Constantino, J.A. de Saja, V. Zucolotto, C. Apetrei, O.N. Oliveira Jr., M.L. Rodriguez-Mendez, Optimized architecture for Tyrosinase-containing Langmuir-Blodgett films to detect pyrogallol, J. Mat. Chem. 21 (2011) 4995.

[28] P. Alessio, F.J. Pavinatto, O.N. Oliveira Jr., J.A. de Saja, C.J.L. Constantino, M.L. Rodriguez-Mendez, Detection of catechol using mixed Langmuir-Blodgett films of a phospholipid and phthalocyanines as voltammetric sensors, Analyst 135 (2010) 2591.

[29] J.H. Zagal, S. Griveau, K.I. Ozoemena, T. Nyokong, F. Bedioui, Carbon nanotubes, phthalocyanines and porphyrins: attractive hybrid materials for electrocatalysis and electroanalysis, Nanosci. Nanotechnol. J. 9 (2009) 2201.

[30] M. Sliwinska, P. Wisniewska, T. Dymerski, J. Namiesnik, W. Wardenci, Food analysis using artificial senses, J. Agric. Food. Chem. 62 (2014) 1423. 
[31] H. Smyth, D. Cozzoino, Instrumental methods (spectroscopy, electronic nose, and tongue) as tools to predict taste and aroma in beverages: advantages and limitations, Chem. Rev. 113 (2013) 1429-1440.

[32] M. Gay, C. Apetrei, I. Nevares, M. del Alamo, J. Zurro, N. Prieto, J.A. de Saja, M.L. Rodriguez-Mendez, Application of an electronic tongue to study the effect of the use of pieces of wood and micro-oxygenation in the aging of red wine, Electrochim. Acta 55 (2010) 6782.

[33] C. Apetrei, I.M. Apetrei, I. Nevares, M. del Alamo, V. Parra, M.L. RodriguezMendez, J.A. de Saja, Using an e-tongue based on voltammetric electrodes to discriminate among red wines aged in oak barrels or aged using alternative methods: correlation between electrochemical signals and analytical parameters, Electrochim. Acta 52 (2007) 2588.

[34] N. Prieto, M. Gay, S. Vidal, O. Aagaard, J.A. de Saja, M.L. Rodriguez-Mendez, Analysis of the influence of the type of closure in the organoleptic characteristics of a red wine by using an electronic panel, Food Chem. 129 (2011) 589.

[35] L. Moreno- Codinachs, J.P. Kloock, M.J. Schoning, A. Baldi, A. Ipatov, A. Bratov, C. Jimenez-Jorquera, Electronic integrated multisensor tongue applied to grape juice and wine analysis, Analyst 133 (2008) 1440.

[36] M. Ghasemi-Varnamkhasti, M.L. Rodriguez-Mendez, S.S. Mohtasebbi, C. Apetrei, J. Lozano, H. Ahmadi, S.H. Razavi, J.A. de Saja, Monitoring the aging of beers using a bioelectronic tongue, Food Control. 25 (2012) 216.
[37] C. Medina-Plaza, G. Revilla, R. Munoz, J.A. Fernandez-Escudero, E. Barajas G. Medrano, J.A. de Saja, M.L. Rodriguez-Mendez, Electronic tongue formed by sensors and biosensors containing phthalocyanines as electron mediators: application to the analysis of red grapes, J. Porphyr. Phthalocyanines 18 (2014) 76.

[38] M. Linaje, M.C. Quintanilla, J.L. del Valle, G. Alcaide, M.L. Rodriguez-Mendez, Improvement of the synthesis of lutetium bisphthalocyanine using the Taguchi method, Analyst 125 (2000) 341.

[39] V. Parra, T. Hernando, M.L. Rodriguez-Mendez, J.A. de Saja, Electrochemical sensor array made from bisphthalocyanine modified carbon paste electrodes for discrimination of red wines, Electrochim. Acta 28 (2004) 51775.

[40] C. Medina-Plaza, J.A. de Saja, M.L. Rodriguez-Mendez, Bioelectronic tongue based on lipidic nanostructured layers containing phenol oxidases and lutetium bisphthalocyanine for the analysis of grapes, Biosens. Bioelectron. 57 (2014) 276.

[41] H. Zhang, Z. Meng, Q. Wang, J. Zheng, A novel glucose biosensor based on direct electrochemistry of glucose oxidase incorporated in biomediated gold nanoparticles-carbon nanotubes composite film, Sens. Actuators B 158 (2011) 23-27.

[42] P. Ribereau-Gayon, Y. Glories, A. Maujean, D. Dubourdieu, in: Handbook of Enology. Vol. 2. The Chemistry of Wine Stabilization and Treatments, John Wiley \& Sons, Chichester (England), 2000 